\title{
Magnetic field modulated exciton generation in organic semiconductors: An intermolecular quantum correlated effect
}

\author{
Baofu Ding (丁宝福), ${ }^{1,2}$ Yao Yao (姚尧), ${ }^{1}$ Xiaoyu Sun (孙晓宇), ${ }^{1}$ Xindong Gao (高歆栋), ${ }^{1}$ Zuoti Xie (谢作提), ${ }^{1}$ \\ Zhengyi Sun (孙正义), ${ }^{1}$ Zijun Wang (王子君), ${ }^{1}$ Xunmin Ding (丁训民), ${ }^{1}$ Yizheng Wu (吴义政), ${ }^{1}$ \\ Xiaofeng Jin (金晓峰), ${ }^{1}$ Wallace C. H. Choy, ${ }^{2}$ Chang-Qin Wu (吴长勤), ${ }^{1, *}$ and \\ Xiaoyuan Hou (侯晓远) $)^{1, \dagger}$ \\ ${ }^{1}$ State Key Laboratory of Surface Physics, Fudan University, Shanghai 200433, China \\ ${ }^{2}$ Electric and Electronic Engineering Department, University of Hong Kong, Hong Kong, China
}

(Received 20 May 2010; published 17 November 2010)

\begin{abstract}
Magnetoelectroluminescence (MEL) of organic semiconductor has been experimentally tuned by adopting blended emitting layer consisting of hole transporting material and electron transporting material. Theory based on Hubbard model fits experimental MEL well, which reveals two findings: (1) spin scattering and spin mixing, respectively, dominate MEL in low-field and high-field region. (2) Blended ratio, and thus the mobility, determines the value of the relative change in the EL in a given magnetic field. Finally successful prediction about the increase in singlet excitons in low field with little change in triplet exciton population further confirms the first finding.
\end{abstract}

DOI: 10.1103/PhysRevB.82.205209

PACS number(s): 71.35.Ji, 72.80.Le, 72.25.Dc, 78.60.Fi

\section{INTRODUCTION}

In the field of organic spintronics, ${ }^{1-13}$ magnetoelectroluminescence (MEL), i.e., magnetic field modulated electroluminescence of organic semiconductor devices with nonmagnetic electrodes, has been widely studied, ${ }^{5-13}$ because it provides a promising way to understand the spin dynamics in organic systems, such as organic semiconductors and biological systems. ${ }^{14}$ Generally, MEL appears to enhance rapidly with magnetic field in the low-field region (typically $<15 \mathrm{mT}$ ) but to saturate or weaken in the high-field region $(>30 \mathrm{mT}) .^{5,6,10,12}$

Unanswered questions include the reason why smaller electric field leads to larger MEL, ,6,8,10,12,15 why insertion of an insulating layer LiF enhances MEL (Ref. 10) and why minority carriers dominate the magnetic field effect in organic semiconductors. ${ }^{6,15-17}$ These form one basic issue, namely what determines the intensity of MEL in a given magnetic field. Theories of intersystem crossing (ISC) (Refs. 9, 10, and 16) or triplet-triplet annihilation (TTA) (Refs. 12 and 13) have been proposed by some groups to qualitatively explain MEL. Both ISC and TTA could be included in a so-called spin mixing (SM) mechanism which occurs only in spin sublevels through spin redistribution. ${ }^{6,14,18-23}$ According to ISC or TTA, the increase in singlet excitons should occur at the expense of triplet excitons, (noticeably, in ISC-based theory, external magnetic field mainly influences the balance of singlet and triplet polaron pair prior to formation of execution. $)^{9,13}$ but Reufer et al. found that no change in singlet-triplet balance occurs, especially in low magnetic field. ${ }^{7}$ Since MEL correlates directly with magnetic field modulated exciton generation, it is critical to consider how magnetic field influences the process of exciton generation. Generally, exciton generation consists of three steps: ${ }^{24}$ (I) carrier injection from electrodes and transport; (II) formation of a hole-electron pair at two adjacent molecules; (III) formation of an exciton at one molecule by hopping. Identifying in which step SM is active and which other mechanism dominates beyond that step are the essential questions that must be addressed. ${ }^{6,18}$ To this end a quantitative explanation of MEL is indispensable.

In this paper, we describe a model formulated by considering the intermolecular correlated effect. Based on the model, the process of magnetic field modulated step (II) and step (III) of exciton generation are quantitatively studied both experimentally and theoretically. It is found that SM only dominates magnetic field modulated step (III) in high magnetic field while spin scattering (SS), in which the redistribution of both spins and charges is involved, ${ }^{25,26}$ controls the modulation of step (II) in low magnetic field. By studying the MEL of the organic light-emitting diode (OLED) with a blended emitting layer, we find that hopping rates of carriers influence both SS and SM. This proves that an intermolecular quantum correlated effect plays an essential role in organic MEL, and suggests that the magnetic field effect in other relevant systems $\mathrm{s}^{3,14,16}$ should be reconsidered.

\section{EXPERIMENT}

For the present work, a special structure of OLED was designed to reveal the effect of intermolecular correlation on excition generation. The structure of OLED with a blended layer is as follows: $\mathrm{ITO} / \mathrm{NPB}(40) / \mathrm{NPB}: \mathrm{Alq}_{3}$ $(20 \mathrm{~nm}) / \mathrm{BCP}(20 \mathrm{~nm}) / \mathrm{Alq}_{3} \quad(50 \mathrm{~nm}) / \mathrm{LiF}(0.8 \mathrm{~nm}) / \mathrm{Al}(100$ $\mathrm{nm})$. Blended layer consisting of tris-(8-hydroxyquinoline) aluminum $\left(\mathrm{Alq}_{3}\right)$ and $\mathrm{N}, \mathrm{N}^{\prime}$-bis(l-naphthyl)- $\mathrm{N}, \mathrm{N}^{\prime}$ -diphenyl-1, 1'-biphentl-4,4'-diamine (NPB), acting as the fluorescent emitting layer, as shown in Fig. 1(a). The device area is approximately $4 \times 4 \mathrm{~mm}^{2}$. Besides the blended layer, $40 \mathrm{~nm} \mathrm{NPB}$ and $50 \mathrm{~nm} \mathrm{Alq}{ }_{3}$ form the hole-transporting and electron-transporting layers, respectively. Because of its wide band gap, ${ }^{27}$ bathocuproine (BCP) is used as an excitonblocking layer to prevent exciton diffusion from the blended layer to the 50-nm-thick $\mathrm{Alq}_{3}$ layer. The anode and composite cathode are, respectively, ITO and $\mathrm{Al} / \mathrm{LiF}$. 
(a)

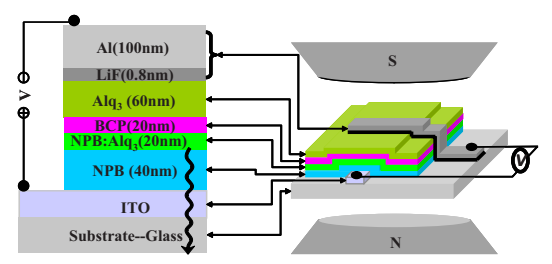

(b)

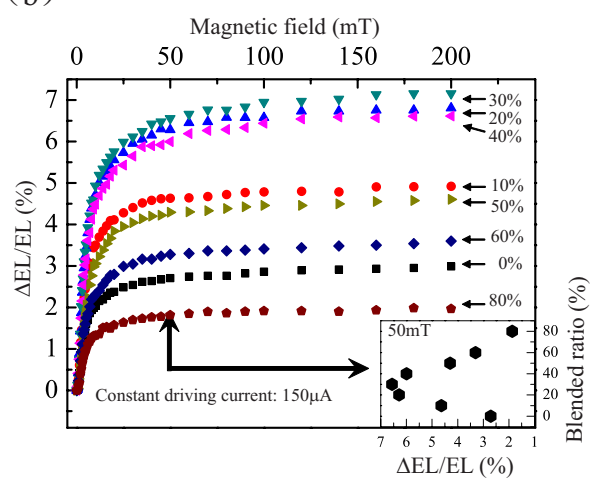

FIG. 1. (Color online) MEL versus blended ratios of NPB to $\mathrm{Alq}_{3}$ of blending layer on MEL. (a) Schematic structure of blending-layer-based OLED. (b) MEL versus blended ratios of NPB to Alq3 of blending layer on MEL under constant current driving of $150 \mu \mathrm{A}$. Inset shows MEL in field of $50 \mathrm{mT}$ versus blended ratios.

Figure 1(b) shows the MEL of eight representative devices with different blending volume ratios of $\mathrm{NPB}$ to $\mathrm{Alq}_{3}$ as a function of external magnetic field under a constant driving current of $150 \mu \mathrm{A}$. The MEL is expressed by $\Delta E L / E L=[(E L(B)-E L(0)] / E L(0)$, where $E L(B)$ and $E L(0)$ are the electroluminescence, respectively, with and without external magnetic field $B$. Similar to the results reported before, ${ }^{6}$ each curve is characterized by fast enhancement in the low field region followed by saturation in the high-field region. The intensity of MEL under a given field depends strongly on the blended ratio. Inset in Fig. 1 shows $\Delta E L / E L$ at $50 \mathrm{mT}$. With the ratio increasing from $0 \%$ to $30 \%$, $\triangle E L / E L$ increases from $2.6 \%$ to $6.8 \%$. However, further increase in the ratio from $30 \%$ to $80 \%$ causes $\triangle E L / E L$ to decrease from $6.8 \%$ to $1.7 \%$. To confirm the relation between MEL and blended ratio, MEL under the other two driving conditions of constant driving voltage $7 \mathrm{~V}$ and constant initial brightness of $20 \mathrm{~cd} / \mathrm{m}^{2}$ show similar behavior. The experiments reveal an intrinsic relation between MEL and the blended ratio. Liu et al. reported previously that electrical conductivity, which is determined by carrier mobility, reaches a minimum around the blended ratio of $30 \%$ in a blended layer of $\mathrm{NPB}$ and $\mathrm{Alq}_{3}{ }^{28}$ The ratio of $30 \%$ agrees well with the ratio in the present experiments at which MEL reaches its maximum. On a quantum scale, mobility of carriers is dominated by their hopping rate. The hopping rate is one of the key factors that affect the interaction among intermolecular carriers, and is intrinsically a type of correlated effect. All of the above experimental results demonstrate that a correlated effect is likely to determine the intensity of MEL.

\section{SIMULATION BASED ON HUBBARD MODEL}

Based on the results shown in Fig. 1(b), it is proposed that the Hubbard model, a typical model for correlated systems, can be used to describe such a correlated effect. ${ }^{29}$ The Hamiltonian, including both the intermolecular transportation of electrons/holes and the interaction between electrons/ holes and nuclear spins, reads,

$$
H=H_{1}+H_{2},
$$

The first part of Eq. (1) includes hopping and Coulomb interaction of carriers,

$$
\begin{aligned}
H_{1}= & -\sum_{i, j, \sigma}\left(t_{i, i^{\prime}}^{h} d_{i, \sigma}^{\dagger} d_{i^{\prime}, \sigma}+t_{j, j^{\prime}}^{e} c_{j, \sigma}^{\dagger} c_{j^{\prime}, \sigma}+\text { H.c. }\right) \\
& +U \sum_{i, j}\left(n_{i \uparrow}^{h} n_{i \downarrow}^{h}+n_{j \uparrow}^{e} n_{j \downarrow}^{e}\right)+V,
\end{aligned}
$$

where $d_{i, \sigma}^{\dagger}\left(d_{i, \sigma}\right)$ and $c_{j, \sigma}^{\dagger}\left(c_{j, \sigma}\right)$ create (annihilate) a hole and an electron with spin $\sigma$ up $(\uparrow)$ or down $(\downarrow)$ in the $i$ th and $j$ th molecule, $i^{\prime}$ and $j^{\prime}$ denote the neighbors of the $i$ th and $j$ th molecule, $t_{i, i^{\prime}}^{h}$ and $t_{j, j^{\prime}}^{e}$ are the hopping rates (in unit of $\mu \mathrm{eV}$ by the uncertainty principle $\Delta E \Delta t \approx \hbar / 2$ ) of hole and electron, $U$ is the Coulomb repulsive energy between two holes or two electrons with different spins at the same molecule, $n_{i, \sigma}^{h}\left(\equiv d_{i, \sigma}^{\dagger} d_{i, \sigma}\right)$ and $n_{j, \sigma}^{e}\left(\equiv c_{j, \sigma}^{\dagger} c_{j, \sigma}\right)$ the corresponding hole and electron number operators, and $V$ the attractive interaction between hole and electron at the same molecule.

The second part of Eq. (1) shows the effect of an external magnetic field and hyperfine interactions,

$$
H_{2}=g \mu_{B} \sum_{i, j}\left[\left(\vec{B}_{\text {ext }}+\vec{B}_{\text {hyp }, i}\right) \cdot \vec{S}_{i}^{h}+\left(\vec{B}_{\text {ext }}+\vec{B}_{\text {hyp }, j}\right) \cdot \vec{S}_{j}^{e}\right],
$$

where $g=2.0$ for organic materials. ${ }^{7} \mu_{B}$ is Bohr magneton, $\vec{B}_{\text {ext }}$ is the external magnetic field chosen to lie in the $z$ direction, $\vec{S}_{i(j)}^{e(h)}$ is the spin of electron (hole) at the $i$ th (jth) molecule and $\vec{B}_{h y p, i(j)}$ is the effective nuclear magnetic field of $i$ th $(j$ th) molecule that will be treated classically. For example, for holes,

$$
\begin{aligned}
\vec{B}_{\text {hyp }, i}^{h} \cdot \vec{S}_{i}^{h}= & B_{\text {hyp }, i}^{h} \cos \theta_{i}^{h}\left(d_{i, \uparrow}^{\dagger} d_{i, \uparrow}-d_{i, \downarrow}^{\dagger} d_{i, \downarrow}\right) \\
& +B_{h y p, i}^{h} \sin \theta_{i}^{h}\left(d_{i, \uparrow}^{\dagger} d_{i, \uparrow}+d_{i, \downarrow}^{\dagger} d_{i, \downarrow}\right),
\end{aligned}
$$

where $\theta_{i}^{h}$ is the angle between $\vec{B}_{h y p, i}$ and hole spin.

In this work, we focus on the intrinsic features of the organic semiconductor. The model is a two-step one including only step (II) and step (III) of exciton generation mentioned above. Generally, hopping rates $t_{i, i^{\prime}}^{h}$ and $t_{j, j^{\prime}}^{e}$ vary from molecule to molecule due to the disorder of organic materials. $t^{h}$ and $t^{e}$ are estimated to be between $1 \mu \mathrm{eV}$ and $100 \mu \mathrm{eV},{ }^{28-33}$ depending on the blended ratio. $U$ is taken as $0.1 \mathrm{eV},{ }^{34}$ which is large enough to prevent double occupancy of the same polarity of carriers at one molecule, yet spin exchange between two molecules still operates when many excitons interacting with each other in step (III) ${ }^{29}$ are considered. To be supplementary, even the changing of $U$ will not affect the result of our theory. So $U$ is a constant. Because of the random orientation of molecular $\vec{B}_{\text {hyp }}$, it is necessary to calculate the statistical average for the angle of 


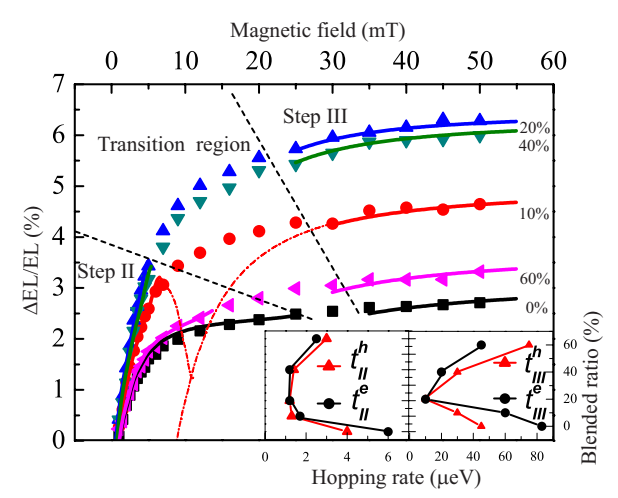

FIG. 2. (Color online) Results of calculation. Dots are experimental data with five representative blended ratios while the solid lines show the relative results of the present two-step model calculation. Two sets of solid lines in low field and high field are results of calculation for the magnetic field modulated step (II) and step (III) of the exciton generation. Dashed-dotted lines are calculated result in transition region. Hopping rate parameters are shown in the insets: $t_{\mathrm{II}}^{h}$ and $t_{\mathrm{II}}^{e}\left(t_{\mathrm{III}}^{h}\right.$ and $\left.t_{\mathrm{III}}^{e}\right)$ are hopping rate of hole and electron, respectively, for magnetic field modulated step (II) [step (III)].

nuclear spins $\theta[\in(0, \pi)]$. Hyperfine interaction $g \mu_{B} B_{h y p, i(j)}$, which plays a basic role in determining magnetic field effect of organic semiconductor, ${ }^{19-23,35,36}$ is set to $0.5 \mu \mathrm{eV},{ }^{34,35,37}$ Thus an external magnetic field about five times higher than the hyperfine interaction $(>25 \mathrm{mT})$ can be considered as a high field. Noticeably, although there are several parameters, the only two variable parameters are hopping rates of $t_{e}$ and $t_{h}$, which will be introduced in the following.

Figure 2 shows results of the present simulation, compared to the corresponding experimental measurements. Without loss of generality, all calculations have been normalized with the result of pure $\mathrm{Alq}_{3}$ structure. Each curve contains two parts corresponding to magnetic field modulated step (II) and step (III), respectively, as marked in Fig. 2. Calculated $\triangle E L / E L$ in the region to which it applies, shown as solid lines, is consistent with the experimental data. It is seen that for step (II), the calculated $\Delta E L / E L$ as shown by solid line increases quickly in low magnetic field, showing close agreement with the experimental results, but weakens as shown by dash dot line representatively for $10 \%$ rather than saturates beyond $5 \mathrm{mT}$ where the hyperfine interaction no longer dominates in transition region. For step (III), the calculated $\triangle E L / E L$ becomes positive at $10 \mathrm{mT}$ as shown by dashed-dotted line in transition region representatively for $10 \%$ and then increases quickly. Following an increase in magnetic field surpassing the nuclear interaction, it saturates and reproduces the experimental data above $25 \mathrm{mT}$ as shown solid line. Two insets in Fig. 2 show the hopping rate parameters for magnetic field modulated step (II) and magnetic field modulated step (III). Hopping rates in step (II) are taken to be one order smaller than those used in step (III) since in step (III), compare to the interaction in the process of hopping to adjacent molecule from remote sites [step (II)]. The shorter distance between electron and hole makes them feel stronger Coulomb attraction in the process of hopping to the same molecule.from the adjacent molecules $[\text { step (III) }]^{38,39}$ It is found that the calculated $\Delta E L / E L$ increases monotonically

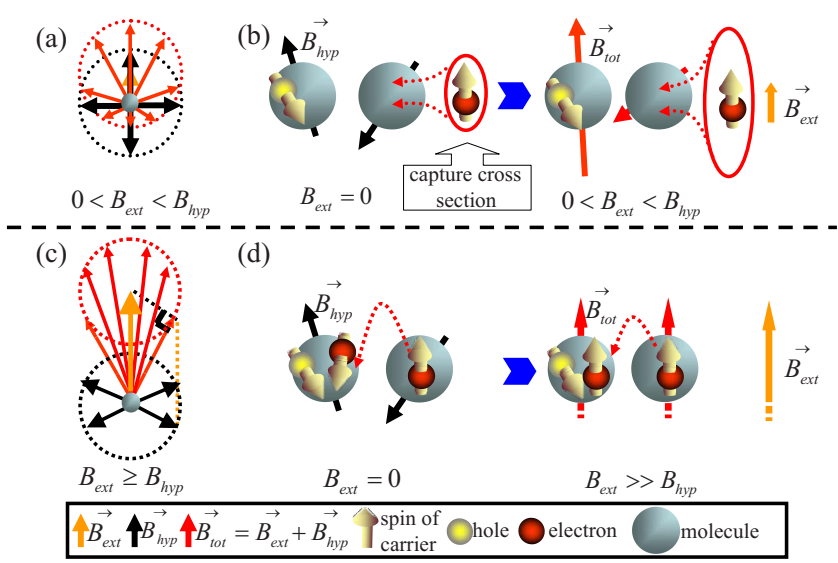

FIG. 3. (Color online) Schematic of the influence of external magnetic field in different ranges on the exciton generation. The light orange (light gray) arrow/black arrow represents external magnetic field $\vec{B}_{\text {ext }}$ / effective nuclear magnetic field $\vec{B}_{\text {hyp }}$, and the red (dark gray) arrow with small arrowhead stands for $\vec{B}_{\text {tot }}$ (the resultant field of $\vec{B}_{\text {ext }}$ and $\vec{B}_{\text {hyp }}$ ). (a) Low $\vec{B}_{\text {ext }}$ changes the uniform distribution of intensity of $\vec{B}_{h y p}$ into nonuniform distribution. (b) Left part indicates that without $\vec{B}_{\text {ext }}$ carrier capture cross section is small. Right part indicates that the nonuniform distribution caused by low $\vec{B}_{e x t}$ increases carrier capture cross section, and thus increases the formation probability of hole-electron pairs. (c) High $\vec{B}_{\text {ext }}$ can align random orientation of $\vec{B}_{\text {hyp }}$ toward the same orientation. (d) Left part shows how singlet hole-electron pair transits to triplet exciton through spin flip without $\vec{B}_{\text {ext }}$. Right part shows when $\vec{B}_{\text {ext }} \gg \vec{B}_{\text {hyp }}$, the spin flip is ineffective, and the singlet hole-electron pair can only transit to singlet exciton.

with decreasing hopping rate in both steps, behaving in the same way as experimentally observed in the inset of Fig. 1(b). In the microscopic view, hopping rate reflect the intensity of interaction between combined carriers. Magnetic field unrelated item of $H_{1}$ in the whole Hamiltonian can dominate the main process of exaction formation if hopping rate is increased. In such case, if hopping rate is small, the intrinsic correlated effect is easy to be perturbed by the external magnetic field. It will be reasonable to observe the strong MEL. To be supplementary, present work only focuses on two regions of MEL, the low- and the high-field region. The transition region will be considered in future. These results confirm that the correlated effect, which is controlled by the intermolecular hopping rate, is essential for MEL and that lowering the hopping rate can enhance the MEL.

\section{DISCUSSION}

To understand the physics of the two-step model a possible explanation is given in Fig. 3, showing schematically how steps (II) and (III) are influenced in different magnetic field regions.

\section{A. Source of MEL in low magnetic field $(<15 \mathrm{mT})$ : Spin-scattering dominated step (III)}

Magnetic field modulated step (II) is shown in Figs. 3(a) and 3(b), demonstrating how a low external magnetic field 
(a)

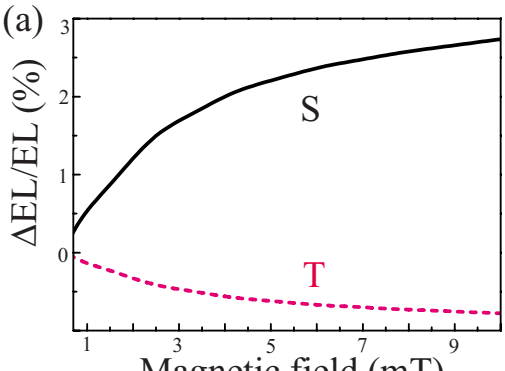

(b)

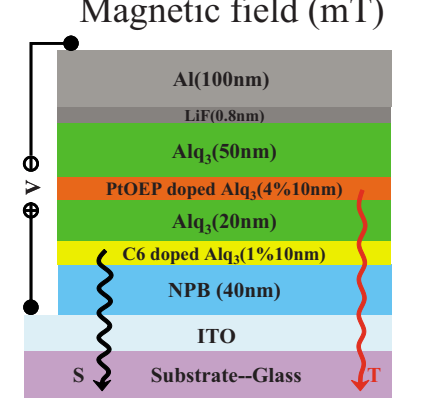

(c)
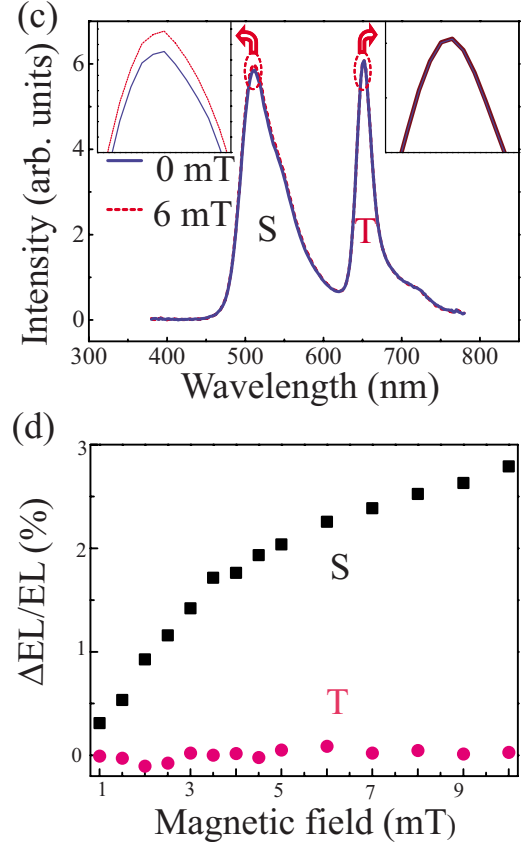

FIG. 4. (Color online) Behaviors of singlet and triplet excitons in the range of low magnetic field where spin scattering dominates. (a) Theoretical prediction of the behavior of singlet and triplet excitons: singlet excitons are more sensitive to magnetic field than triplet ones. Black solid and red (dark gray) dash lines are for singlet and triplet excitons, respectively. (b) Schematic architecture of devices consisting of a layer doped with fluorescent dye C6 for detecting and removing singlet excitons and another layer doped with a phosphorescent dye for detecting triplet excitons. (c) Spectra of OLED simultaneously emitting fluorescence and phosphorescence with and without $6 \mathrm{mT}$ field. Left and right insets show amplified curves around two peaks. (d) Experimental results for behaviors of singlet and triplet excitons in low magnetic field.

influences the SS, and thus increases the generation probability of hole-electron pairs. If no external magnetic field $\vec{B}_{\text {ext }}$ exists, the intensity value of $\vec{B}_{\text {hyp }}$, the effective nuclear magnetic field of a molecule, is identical for each molecule, as shown by black arrows in Fig. 3(a). This means that the intensity distribution of $B_{h y p}$ is uniform in space. However, for a given low $\vec{B}_{e x t}$, the total $\vec{B}_{t o t}$ of $\vec{B}_{e x t}$ and $\vec{B}_{h y p}$ is not identical due to the random orientation of $\vec{B}_{\text {hyp }}$, resulting in nonuniform intensity distribution of $B_{t o t}$ as shown by red (dark gray) arrows with small arrowhead in Fig. 3(a). If $B_{\text {ext }} \gg B_{h y p}$, all $\vec{B}_{t o t}$ are almost the same as $\vec{B}_{\text {ext }}$ and the intensity distribution of $\vec{B}_{t o t}$ should be uniform. The nonuniform $\vec{B}_{t o t}$ acts like disorder in an energy distribution for the carrier spins, strengthening their scattering and resulting in enhancement of the formation probability of hole-electron pairs. The dependence of spin scattering on $B_{\text {ext }}$ is very similar to that in the case of dilute magnetic semiconductors, where the spin scattering between carrier and magnetic atom is proportional to $x(1-x)$, where $x$ is the density of magnetic atoms. ${ }^{26}$ In Fig. 3(b), a carrier capture cross section is used to describe the spin scattering. Without $\vec{B}_{e x t}$, the intensity distribution of $\vec{B}_{h y p}$ is uniform. The carrier capture cross section for a molecule is small, as shown in the left part of Fig. 3(b). Low $\vec{B}_{\text {ext }}$ will cause nonuniform intensity distribution of $\vec{B}_{t o t}$, and thus enlarge the carrier capture cross section in the right part of Fig. 3(b). When $B_{\text {ext }} \gg B_{\text {hyp }}$, the intensity distribution of $\vec{B}_{t o t}$ is once again uniform and the carrier capture cross section will decrease to the value in the case of $B_{\text {ext }}=0$.

\section{B. Source of MEL in low magnetic field ( $>25 \mathrm{mT})$ : Spin-mixing dominated step (II)}

Magnetic field modulated step (III) is shown in Figs. 3(c) and 3(d), demonstrating how a high external magnetic field suppresses the spin flip, and thus increases the ratio of singlet to triplet excitons. In the case of $B_{e x t}=0$, as shown in the left part of Fig. 3(d), a hole and an electron are located at two adjacent molecules to form a singlet hole-electron pair. When the electron hops to the adjacent molecule where the hole resides, the electron spin will be modulated by both $\vec{B}_{h y p}^{h}$ and $\vec{B}_{\text {hyp }}^{e}$ of the two molecules where hole and electron, respectively, reside. Due to their random orientation, $\vec{B}_{h y p}^{h}$ and $\vec{B}_{h y p}^{e}$ point to different directions. In such case, $\vec{B}_{h y p}^{e}$ provides the transversal component to $\vec{B}_{h y p}^{h}$. It is well known that the transversal component makes spin flip feasible during electron hopping. ${ }^{26}$ Therefore the singlet hole-electron pair can undergo transition to a triplet exciton. This is indeed the mechanism of SM caused by random nuclear field. ${ }^{40}$ However, a high $B_{e x t}$ can align $B_{t o t}$ toward $B_{e x t}$, therefore $B_{t o t}$ would no longer be random as $B_{h y p}$, as shown by red (dark gray) arrows in Fig. 3(d). The right part of Fig. 3(d) shows how when $B_{\text {ext }} \gg B_{\text {hyp }}$, compared to the large longitudinal component of $\vec{B}_{t o t}$ the transversal component becomes insignificant. In this case spin flip will hardly occur. Therefore the inhibition of SM effectively increases the number of singlet excitons. MEL in different regions of magnetic field is dominated by different mechanisms-SS and SM. They dominate the magnetic field modulated step (II) and step (III), respectively. 


\section{THEORETICAL PREDICTION AND EXPERIMENTAL CONFIRMATION ON CHANGE IN SINGLET AND TRIPLET EXCITON IN LOW MAGNETIC FIELD}

From the two-step model, the intrinsic property of the mechanism of SS is that an increase in singlet excitons is not obtained solely at the expense of triplet excitons. Figure 4(a) shows the theoretical result concerning the variation in singlet and triplet excitons with low magnetic field, and it can be seen that the influence of magnetic field on singlet excitons is much stronger than that on triplet excitons. Experimentally, Reufer et al. have examined whether an external magnetic field could change the singlet/triplet ratio by using a phosphorescent hydrocarbon polymer with simultaneous emission of fluorescence and phosphorescence, but the method could hardly prevent the internal energy transfer between singlet and triplet excitons. ${ }^{7,18}$ In order to eliminate the internal energy transfer, a special structure of OLED was adopted, which was similar to the structure reported before for studying the phosphorescence of phosphorescent dye 2,3,7,8,12,13,17,18-octaethyl-21 H,23Hporphine platinum (II) $(\mathrm{PtOEP}) .{ }^{41} \mathrm{ITO} / \mathrm{NPB}(40 \mathrm{~nm}) / \mathrm{Alq}_{3}$ doped with $\mathrm{C} 6(1 \% 10 \mathrm{~nm}) / \mathrm{Alq}_{3}(20 \mathrm{~nm}) / \mathrm{Alq}_{3}$ doped with $\operatorname{PtOEP}(4 \% 10 \mathrm{~nm}) / \mathrm{Alq}_{3}(50 \mathrm{~nm}) / \mathrm{LiF} / \mathrm{Al}$ shown in Fig. 4(b) C6 stands for Coumarine 540. Since C6 (PtOEP) is a fluorescent (phosphorescent) dye with efficient Förster (Dexter) energy transfer from singlet (triplet) exciton of $\mathrm{Alq}_{3}$ to singlet (triplet) exciton of C6 (PtOEP), C6 (PtOEP) doped $\mathrm{Alq}_{3}$ layer with volume doping ratio of $1.0 \%(4.0 \%)$ acts as the singlet (triplet) exciton recombination zone. The singlet exciton recombination zone and the triplet exciton recombination zone are separated by a $20 \mathrm{~nm} \mathrm{Alq}$ layer. $20 \mathrm{~nm}$ is sufficiently thick to avoid internal energy transfer between the two recombination zones. ${ }^{34}$ Thus the variation in singlet and triplet excitons in magnetic field should be observed simultaneously. Figure 4(c) shows the MEL of the device under a field of $6 \mathrm{mT}$. The peaks of $510 \mathrm{~nm}$ and $652 \mathrm{~nm}$ are emitted from the singlet exciton recombination zone and the triplet exciton recombination zone, respectively. The spectra adjacent to the two peaks are amplified and shown in the left and right insets. With a field of $6 \mathrm{mT}, \Delta E L / E L$ around 510 $\mathrm{nm}$ is calculated to be about $2.2 \%$ while $\Delta E L / E L$ around 652 $\mathrm{nm}$ is almost zero. Figure $4(\mathrm{~d})$ shows the experimental results of $\Delta E L / E L$ for singlet excitons and triplet excitons in the low field range. Increasing the field from 1 to $10 \mathrm{mT}$, $\triangle E L / E L$ for singlet excitons increases from $0.2 \%$ to $2.8 \%$ while $\Delta E L / E L$ for triplet excitons is nearly zero over this range. Similar to the theoretical results shown in Fig. 4(a) these experimental results also indicate that the influence of a low magnetic field on singlet excitons is much stronger than that on triplet excitons. Some difference exists between the theoretical result shown in Fig. 4(a) and the experimental result shown in Fig. 4(d) for the variation in triplet excitons. It means more physical processes should be taken into consideration in the model. Furthermore, it must be mentioned that the structure of OLED cannot be used experimentally to observe the behavior of triplet excitons in high magnetic field, since a high field influences many unclear effects in PtOEP, such as spin-orbit interaction and spin-spin interaction, which in turn influence the experimental result.

\section{CONCLUSION}

In conclusion, we report that MEL is strongly dependant on the blended ratio of NPB to $\mathrm{Alq}_{3}$. The maximum MEL occurs at the blended ratio of $30 \%$, which corresponds to the minimum conduction of blended layer. Compared to the theoretical result, we confirm that the intermolecular and intramolecular correlations play the essential role in MEL. In addition, to confirm the result as predicted by our model, we adopt the special structure of OLED to avoid internal energy transfer between singlet and triplet exciton, so as to observe them simultaneously and individually. The increase in singlet excitons in low field with little change in triplet exciton population exceeds all experimental and theoretical results before. Our finding, that magnetoelectroluminescence in low/high field can reflect electron-hole pair/exciton formation, strongly indicates that magnetic field effect can be used as a tool to reveal the carrier-photon conversion process in organic optoelectronic devices.

\section{ACKNOWLEDGMENTS}

This work is supported by the National Natural Science Foundation of China and Shanghai Science and Technology Commission (Grant No. 08JC1402300) and the MST of China (Grant No. 2009CB929200). B.F.D. acknowledges HKU and the support of the Grant No. HKU712108 from the Research Grants Council of the HK Special Administrative Region, China. W.C.H.C. thanks UGC (Grant No. 400897) of the University of Hong Kong and Hong Kong Research Grants Council (Grant No. HKU712108). Y.Y. and C.Q.W. are also supported by the EC Project of OFSPIN (Grant No. NMP3-CT-2006-033370). We would like to thank Edward Obbard for his assistance in proofreading the manuscript.

\footnotetext{
*cqw@fudan.edu.cn

†xyhou@fudan.edu.cn

${ }^{1}$ V. Dediu, M. Murgia, F. C. Matacotta, C. Taliani, and S. Barbanera, Solid State Commun. 122, 181 (2002).

${ }^{2}$ V. Dediu, L. E. Hueso, I. Bergenti, and C. Taliani, Nature Mater. 8, 707 (2009).

${ }^{3}$ Z. H. Xiong, D. Wu, Z. V. Vardeny, and J. Shi, Nature (London)
}

427, 821 (2004).

${ }^{4}$ B. F. Ding, Y. Q. Zhan, Z. Y. Sun, X. M. Ding, X. Y. Hou, Y. Z. Wu, I. Bergenti, and V. Dediu, Appl. Phys. Lett. 93, 183307 (2008).

${ }^{5}$ J. Kalinowski, M. Cocchi, D. Virgili, P. Di Marco, and V. Fattori, Chem. Phys. Lett. 380, 710 (2003).

${ }^{6}$ F. J. Wang, H. Bässler, and Z. Valy Vardeny, Phys. Rev. Lett. 
101, 236805 (2008).

${ }^{7}$ M. Reufer, M. J. Walter, P. G. Lagoudakis, A. B. Hummel, J. S. Kolb, H. G. Roskos, U. Scherf, and J. M. Lupton, Nature Mater. 4, 340 (2005).

${ }^{8}$ Y. Wu, Z. H. Xu, B. Hu, and J. Howe, Phys. Rev. B 75, 035214 (2007).

${ }^{9}$ J. Kalinowski, M. Cocchi, D. Virgili, V. Fattori, and P. Di Marco, Phys. Rev. B 70, 205303 (2004).

${ }^{10}$ H. Odaka, Y. Okimoto, T. Yamada, H. Okamoto, M. Kawasaki, and Y. Tokura, Appl. Phys. Lett. 88, 123501 (2006).

${ }^{11}$ J. Wilkinson, A. H. Davis, K. Bussman, and J. P. Long, Appl. Phys. Lett. 86, 111109 (2005).

${ }^{12}$ R. Liu, Y. Zhang, T. L. Lei, P. Chen, and Z. H. Xiong, J. Appl. Phys. 105, 093719 (2009).

${ }^{13}$ B. Hu, L. Yan, and M. Shao, Adv. Mater. 21, 1500 (2009).

${ }^{14}$ K. Maeda, K. B. Henbest, F. Cintolesi, I. Kuprov, C. T. Rodgers, P. A. Liddell, D. Gust, C. R. Timmel, and P. J. Hore, Nature (London) 453, 387 (2008).

${ }^{15}$ T. D. Nguyen, Y. Sheng, J. Rybicki, and M. Wohlgenannt, Phys. Rev. B 77, 235209 (2008).

${ }^{16}$ B. Hu and Y. Wu, Nature Mater. 6, 985 (2007).

${ }^{17}$ F. L. Bloom, W. Wagemans, M. Kemerink, and B. Koopmans, Phys. Rev. Lett. 99, 257201 (2007).

${ }^{18}$ J. M. Lupton and C. Boehme, Nature Mater. 7, 598 (2008).

${ }^{19}$ N. E. Geacintov, M. Pope, and S. Fox, J. Phys. Chem. Solids 31, 1375 (1970).

${ }^{20}$ C. D. Buckley and K. A. McLauchlan, Mol. Phys. 54, 1 (1985).

${ }^{21}$ E. L. Frankevich, I. A. Sokolik, and A. A. Lymarev, Mol. Cryst. Liq. Cryst. 175, 41 (1989).

${ }^{22}$ R. P. Groff, R. E. Merrifield, A. Suna, and P. Avakian, Phys. Rev. Lett. 29, 429 (1972).

${ }^{23}$ P. W. Adams, Phys. Rev. Lett. 92, 067003 (2004).

${ }^{24}$ M. Segal, M. Singh, K. Rivoire, S. Difley, T. Van Voorhis, and M. A. Baldo, Nature Mater. 6, 374 (2007).

${ }^{25}$ M. Viret, D. Vignoles, D. Cole, J. M. D. Coey, W. Allen, D. S. Daniel, and J. F. Gregg, Phys. Rev. B 53, 8464 (1996).
${ }^{26}$ M. Foygel, J. Niggemann, and A. G. Petukhov, IEEE Trans. Magn. 43, 3040 (2007).

${ }^{27}$ I. G. Hill and A. Kahn, J. Appl. Phys. 86, 4515 (1999).

${ }^{28}$ S. W. Liu, J. H. Lee, C. C. Lee, C. T. Chen, and J. K. Wang, Appl. Phys. Lett. 91, 142106 (2007).

${ }^{29}$ A. Auerbach, Interacting Electrons and Quantum Magnetism (Springer, New York, 1998).

${ }^{30}$ A. J. Drew, F. L. Pratt, J. Hoppler, L. Schulz, V. Malik-Kumar, N. A. Morley, P. Desai, P. Shakya, T. Kreouzis, W. P. Gillin, K. W. Kim, A. Dubroka, and R. Scheuermann, Phys. Rev. Lett. 100, 116601 (2008).

${ }^{31}$ T. Y. Chu and O. K. Song, J. Appl. Phys. 104, 023711 (2008).

${ }^{32}$ S. L. M. van Mensfoort, V. Shabro, R. J. de Vries, R. A. J. Janssen, and R. Coehoorn. J. Appl. Phys. 107, 113710 (2010).

${ }^{33}$ Y. Nagata and C. Lennartz, J. Chem. Phys. 129, 034709 (2008).

${ }^{34}$ P. A. Bobbert, T. D. Nguyen, F. W. A. van Oost, B. Koopmans, and M. Wohlgenannt, Phys. Rev. Lett. 99, 216801 (2007).

${ }^{35}$ T. D. Nguyen, G. H. Markosian, F. Wang, L. Wojcik, X. G. Li, E. Ehrenfreund, and Z. V. Vardeny, Nature Mater. 9, 345 (2010).

${ }^{36}$ V. Dyakonov and E. Frankevich, Chem. Phys. 227, 203 (1998).

${ }^{37}$ Y. Sheng, D. T. Nguyen, G. Veeraraghavan, Ö. Mermer, M. Wohlgenannt, S. Qiu, and U. Scherf, Phys. Rev. B 74, 045213 (2006).

${ }^{38}$ M. Kuwata-Gonokami, N. Peyghambarian, K. Meissner, B. Fluegel, Y. Sato, K. Ema, R. Shimano, S. Mazumdar, F. Guo, T. Tokihiro, H. Ezaki, and E. Hanamura, Nature (London) 367, 47 (1994).

${ }^{39}$ D. J. Styers-Barnett, S. P. Ellison, B. P. Mehl, B. C. Westlake, R. L. House, C. Park, K. E. Wise, and J. M. Papanikolas, J. Phys. Chem. C 112, 4507 (2008).

${ }^{40} \mathrm{~W}$. Barford and E. E. Moore, arXiv:physics/0410253 (unpublished).

${ }^{41}$ M. A. Baldo, D. F. O’Brien, Y. You, A. Shoustikov, S. Sibley, M. E. Thompson, and S. R. Forrest, Nature (London) 395, 151 (1998). 